Ann. Zootech., 1985, 34 (1), 31-48

\title{
Production et morbidité des lapines reproductrices I. - Effets du rationnement alimentaire
avant et pendant la première gestation (1)
}

\author{
P. COUDERT, F. LEBAS * \\ I.N.R.A., Station de Pathologie aviaire et de Parasitologie \\ Centre de Tours, B.P. 1, Nouzilly, F $37 j 80$ Monnaie \\ *I.N.R.A., Laboratoire de Recherches sur l'élevage du Lapin \\ Centre de Toulouse, B.P. 27, F 31326 Castanet-Tolosan Cedex
}

\begin{abstract}
Résumé
A l'âge de 79 jours, 124 lapines de type croisé I.N.R.A. A 1067 (Néozélandais Blanc $\times$ Californien) ont été réparties entre les 4 traitements du schéma factoriel $2 \times 2$ correspondant à une alimentation rationnée $(150 \mathrm{~g} / \mathrm{j}$ ) oul à volonté (environ $200 \mathrm{~g} / \mathrm{j}$ ) de lâge de 79 jours à la première mise bas avec inversion ou non de niveau d'alimentation à partir du $13^{\text {" }}$ jour de gestation (diagnostic de gestation positif). Les femelles ont été représentées au mâle 10 jours après chaque mise bas et suivies au cours des 3 premières portées.

Le rationnement initial a réduit de 11 p. 100 le poids vif des lapines mesuré lors de la première saillie. Par contre, il n'altère pas le poids adulte estimé d'après le poids mesuré 7 jours après chacune des mises bas. Le niveau de rationnement en fin de $1^{\text {re }}$ gestation n'a qu'un effet transitoire sur le poids vif des lapines.

Les lapines rationnées lors de leur $1^{\text {re }}$ fécondation ont donné naissance ì des premières portées d'effectif significativement réduit : 8,35 vs 9,88 nés vivants/mise bas. Par contre, ce phénomène n'est plus observé lors des mises bas ultérieures. Le rationnement des lapines durant les deux derniers tiers de la $1^{\text {r" }}$ gestation n'a entraîné aucune altération significative du poids moyen des lapereaux lors de la $1^{\text {" }}$ mise bas : $52,9 \mathrm{~g}$ vs $51,6 \mathrm{~g}$ pour les Iapines alimentées à volonté. Pàr contre, ce rationnement a entraîné une fonte du cheptel plus précoce. Enfin, le changement du niveau d'alimentation au cours de la $1^{\text {rn }}$ gestation a eu des conséquences néfastes en $2^{\prime \prime}$ et $3^{\prime \prime}$ portée d'une part sur la mortalité des lapereaux allaités, d'autre part sur la fonte du cheptel. Au cours des 22 semaines d'expérimentation séparant la $1^{\text {re }}$ présentation au mâle des femelles, derniers sevrages pris en compte, le groupe des 31 lapines initiales ayant toujours été alimenté à volonté, a permis le scvrage de 636 lapereaux, alors que pour chacun des 3 autres groupes, ce nombre n'a été que de 529 à 539 lapereaux.
\end{abstract}

Mots clés : lapin, femelle, gestation, restriction alimentaire, morbidité.

\section{Introduction}

Le rationnement des jeunes lapines avant ou pendant la première gestation, prôné par les uns, déconseillé par les autres, a fait l'objet de peu de travaux de recherche,

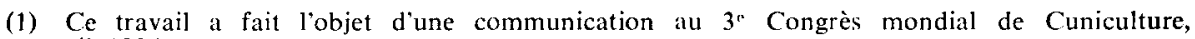
Rome, avril 1984. 
et ceux-ci ne concernent généralement que la première portée. Ainsi, VAN DEN BroEcK \& Lampo (1977 et 1979), Hulot, Mariana \& Lebas (1982) ont montré qu'un rationnement à 50 p. 100 ou 75 p. 100 du niveau de consommation spontanée pratiqué avant la première présentation au mâle, réduit la précocité sexuelle des lapines. Selon Hulot, Mariana \& Lebas (1982), ce rationnement réduit également la taille de la portée. D'autre part, Viard-Drouet, Provot \& Coudert (1984) ont montré qu'un rationnement des jeunes lapines aux environs de 75 p 100 ne modifie pas leur profil biochimique sanguin avant ou pendant la première gestation. Enfin, LeBAS (1975) a montré que le rationnement des lapines durant la 2" gestation, suivi d'une alimentation à volonté dès la mise bas, favorise nettement la production laitière des 8 à 10 premiers jours, sans altération de la taille de portée à la naissance. Ce dernier résultat a été indûment extrapolé aux premières gestations et a conduit nombre d'éleveurs à rationner leurs futures reproductrices en espérant bénéficier de l'accroissement de production laitière.

11 nous est donc apparu intéressant d'étudier l'incidence d'un rationnement précoce, à partir de 11 semaines d'âge, sur les performances et la morbidité des lapines, observées dans un laps de temps correspondant aux 3 premières poriées. En outre, nous avons voulu connaître l'incidence propre du rationnement sur la productivité ultérieure et la longévité des lapines lorsque ce rationnement n'est appliqué que durant les 2 derniers tiers de la gestation, au moment où les besoins de gestation et de fin de croissance de la mère peuvent entrer en compétition.

\section{Matériel et méthodes}

\section{A. Protocole expérimental}

Du sevrage à l'âge de 79 jours, des jeunes lapines sont alimentées ad libitum. A cet âge, elles sont réparties en 4 lots égaux correspondant au schéma factoriel $2 \times 2$ :

$1^{\text {"r }}$ lot RR : 31 femelles rationnées à $150 \mathrm{~g} / \mathrm{j}$ jusqu'à la première mise bas,

2" lot RA : 31 femelles rationnées jusqu'à la première palpation positive (13" jour de gestation) puis alimentées ad libitum.

3" lot AR : 31 femelles nourries ad libitum jusqu'à la première palpation positive, puis rationnées à $150 \mathrm{~g} / \mathrm{j}$ jusqu'à la première mise bas,

$4^{\text {e }}$ lot AA : 31 femelles toujours nourries ad libitum.

A partir de la première mise bas et jusqu'à la fin de l'essai, toutes les femelles sont nourries à volonté.

Les effets de ces 4 traitements sont évalués sur le poids des lapines, leurs performances zootechniques, et sur limportance et la nature de la fonte du cheptel à l'issue du sevrage de la 3" portée. L'essai est arrêté à la suite du sevrage des portées nées 22 semaines après les premières saillies. Celles-ci ont lieu lorsque les femelles ont atteint l'âge de 123 jours. 


\section{B. Locaux et matériel}

Les femelles sont logées et également réparties dans 2 cellules d'ćlevage identiques au domaine I.N.R.A. du Magneraud. Ces cellules donnant dans un même sas, sont ventilées et chauffécs en hiver par air pulsé. Le thermostat est réglé à $17^{\circ} \mathrm{C}$. Dans chaque cellule, les cages sont réparties sur les deux faces d'une batterie à 3 étages. Mâles et femelles sont équitablement répartis sur l'ensemble. Les boîtes à nid sont en tôle galvanisée à fond en bois, et litières en copeaux de bois dépoussiéré. L'abreuvement se fait par des pipettes automatiques. Avant l'entrée des femelles dans chacune des cellules, le local et le matériel sont nettoyés et désinfectés à la vapeur d'eau sous pression, puis à la vapeur de formol. Au cours de la production, chaque cellule est éclairée $16 \mathrm{~h} / 24$ heures.

\section{Animaux}

Les femelles de la souche I.N.R.A. 1067, nées les 5 et 6 décembre 1980 ont été achetées à un multiplicateur local à l'âge de 56 jours. Les mâles sont d'une lignée de Néozélandais Blanc sélectionnés pour les aptitudes bouchères : ils sont de 3 semaines plus âgés que les femelles. Les 160 femelles achetées sont placées 6 par cage à leur arrivée ( $56 \mathrm{j}$.), puis 3 par cage à partir de 79 jours et enfín 1 par cage à partir de 107 jours. A cet âge, les femelles dont le poids est inférieur à la moyenne moins 1 écart type sont éliminées à l'intérieur de chacune des deux sous-populations « rationnement» et "ad libitum». A ce moment, sont également éliminées les fomelles présentant des signes de morbidité (mal de pattes, torticoli, malocelusion dentaire, ...). Pour chacune des deux sous-populations, un même nombre de lapines est éliminé.

\section{Aliment}

L'aliment employé durant tout l'essai est fourni par une firme commerciale ; il contient 17,0 p. 100 de protéines brutes et 13,5 p. 100 de cellulose brute. La consommation spontanée entre l'âge de 80 jours et la première misc bas a été antérieurement estimée. Elle est régulière durant cette période et égale à $200 \mathrm{~g} /$ jour avec ce type d'aliment. Le rationnement pratiqué dans la présente expérimentation se situe donc à 75 p. 100 de la consommation spontanée.

\section{E. Prophylaxie et suivi sanitaire}

Aucune prophylaxic médicale, ni aucun traitement ne sont effectués durant l'essai ; les seules mesures prises correspondent à la prophylaxie hygiénique. Ainsi, après chaque sevrage, la cage et la boîte à nid sont remplacécs par du matériel nettoyé et désinfecté à la vapeur d'eau sous pression. Les femelles n'ont accès à la boîte à nid que 3 jours avant la date prévue pour la mise bas. L'état sanitaire de chaque femelle est évalué cliniquement, au moins à l'occasion de chaque palpation (diagnostic de gestation) et de chaque mise bas. Les femelles présentant les signes cliniques d'un début d'affection pathologique (coryza avec jetage, abcès mammaire ou cutané, torticoli, maigreur, ...) sont éliminées dès le sevrage de la portće en cours. Toutes les femelles mortes ou éliminées sont autopsiées. 


\section{F. Gestion zootechnique du troupeau}

La gestion, entièrement suivie par informatique, correspond au rythme hebdomadaire suivant :

- vendredi : saillie des femelles, après choc lumineux pour les prenières présentations (passage de $8 \mathrm{~h}$ à $16 \mathrm{~h} / 24 \mathrm{~h}, 5$ jours avant). Ensuite, les femelles sont présentées au mâle 10 jours au plus tôt après la mise bas ;

- Jundi et mardi : mises bas ;

- mercredi : sevrage à l'âge de 29-30 jours ;

— jeudi : palpation des femelles, 13 jours après la saillie. Les femelles détectées vides sont représentées au mâle le lendemain.

Les femelles qui refusent l'accouplement un vendredi sont représentées au mâle le vendredi suivant. Elles sont éliminées pour «stérilité » après 3 saillies infécondes, deux refus d'accouplement étant équivalents à une saillie inféconde.

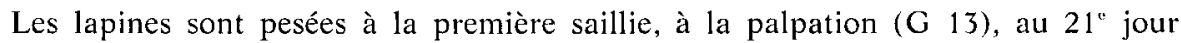
de gestation ( $\mathrm{G} \mathrm{21}$ ), à la mise bas $(\mathrm{MB})$ et 7 jours après mise bas ( $\mathrm{L} 7$ ). Dès la mise bas constatée, les lapereaux morts et vivants sont dénombrés et les vivants sont pesés ensemble (poids de portée naissance). Le jour du sevrage, les lapereaux sont à nouveau dénombrés et pesés ensemble.

1 L'ensemble des données quantitatives et qualitatives (état sanitaire, ...) relatives à chaque femelle est régulièrement enregistré sur un fichier unique en vue de l'exploitation statistique ultérieure.

\section{G. Analyse mathénatique des données}

Toutes les données numériques sont étudiées selon un schéma d'analyse de variance factoriel $2 \times 2$ avec interaction, selon le programme ANVAR pour effectifs déséquilibrés (Bachacou, Masson, Millier, 1981) au Centre de calcul I.N.R.A. de Toulouse. Les comparaisons de proportions sont faites par test de $\chi^{2}$ de Pearson.

\section{Résultats}

A. Poids de femelles anant la première saillie

Les poids à 79 jours des femelles sont comparables pour les 4 lots : 2332 ㅍ. $230 \mathrm{~g}$. Le rationnement à $150 \mathrm{~g} / \mathrm{j}$ à partir de 79 jours réduit significativement la vitesse de croissance : $18,0 \mathrm{~g} / \mathrm{j}$ dans les 2 lots rationnés, contre 28,1 pour les 2 lots nourris à volonté. A 123 jours, lors des premières présentations au mâle, le poids des femelles des deux lots rationnés est inférieur de 11 p. 100 à celui des lapines alimentées à volonté. 
B. Des premiers accouplements à la première palpation positive

Dans le groupe des femelles rationnées ( $R R+R A), 5$ individus ont été éliminés entre l'âge de 123 jours et la palpation positive : 3 pour stérilité et 2 pour torticoli. Par contre, dans le groupe alimenté ad libitum (AR + AA), aucune lapine n'a dû être éliminée, mais 2 sont mortes non fécondées dans les 10 jours qui suivirent leur saillie. La répartition des effectifs de femelles à partir de l'âge de 123 jours figure au tableau 1. Durant cette période, le gain de poids des animaux alimentés ad libitum est 2,5 fois plus important $(\mathrm{P}<0,01)$ que celui des animaux rationnés : $+292 \mathrm{~g} v \mathrm{~s}+116 \mathrm{~g}$.

\section{Tableau 1}

Effectif des femelles vivantes aux différents stades de production et ventilation des lapines disparues.

Number of live and dead females at the various stages of production.

\begin{tabular}{|c|c|c|c|c|c|c|c|c|}
\hline \multirow{2}{*}{$\begin{array}{l}\text { Lots } \\
\text { Groups }\end{array}$} & \multicolumn{6}{|c|}{$\begin{array}{c}\text { Lapines vivantes } \\
\text { Live does }\end{array}$} & \multicolumn{2}{|c|}{$\begin{array}{l}\text { Cumul des causes } \\
\text { de disparition } \\
\text { Cumulated causes } \\
\text { of disappearance }\end{array}$} \\
\hline & $\begin{array}{l}\text { Départ } \\
\text { Initial } \\
\text { number }\end{array}$ & $\begin{array}{r}\text { Palpation } \\
+\left(\begin{array}{l}G \\
13\end{array}\right)\end{array}$ & MB 1 & MB 2 & $\begin{array}{c}\text { MB } 3 \\
\text { (1) }\end{array}$ & $\begin{array}{l}\text { Fin essai } \\
\text { End of } \\
\text { the trial }\end{array}$ & $\begin{array}{l}\text { Mort } \\
\text { Death }\end{array}$ & $\begin{array}{l}\text { Elimi- } \\
\text { nation }\end{array}$ \\
\hline \multirow{2}{*}{$\begin{array}{l}\text { RR } \\
\text { RA }\end{array}$} & \multirow{2}{*}{62} & 29 & 29 & 24 & $18+4$ & 22 & 3 & 6 \\
\hline & & 28 & 27 & 25 & $18+5$ & 18 & 4 & 9) \\
\hline \multirow{2}{*}{$\begin{array}{l}\text { AR } \\
\text { AA }\end{array}$} & \multirow{2}{*}{62} & 30 & 29 & 24 & $16+4$ & 17 & 9 & 5 \\
\hline & & 30 & 30 & 27 & $19+7$ & 21 & 4 & 6 \\
\hline
\end{tabular}

(1) Nombre de femelles ayant fait 3 mises bas + femelles vivantes au même moment mais n'ayant fait que 2 mises bas.

Number of females having littered three times + live females having littered only twice.

\section{De la palpation positive à la première mise bas}

Au cours de cette période, 2 lapines sont mortes, l'une au 22" jour de gestation dans le lot AR et l'autre au $30^{\circ}$ jour de gestation dans le lot RA. Durant cette période, l'effet principal observé sur les variations de poids de femelles (fig. 1) est celui d niveau d'alimentation de la période précédente. Cela est particulièrement net pendant les 8 jours suivant le changement de régime. En particulier, avec le même niveau d'alimentation de $150 \mathrm{~g} /$ jour, les lapines du lot $R R$ ont un gain de poids 
de $352 \mathrm{~g}$, alors que celui des lapines AR n'est que de $75 \mathrm{~g}$. Ainsi, le niveau d'alimentation durant cette période a-t-il un effet seulement comme facteur d'amplification du gain de poids entre la palpation positive et le $21^{\circ}$ jour de gestation.

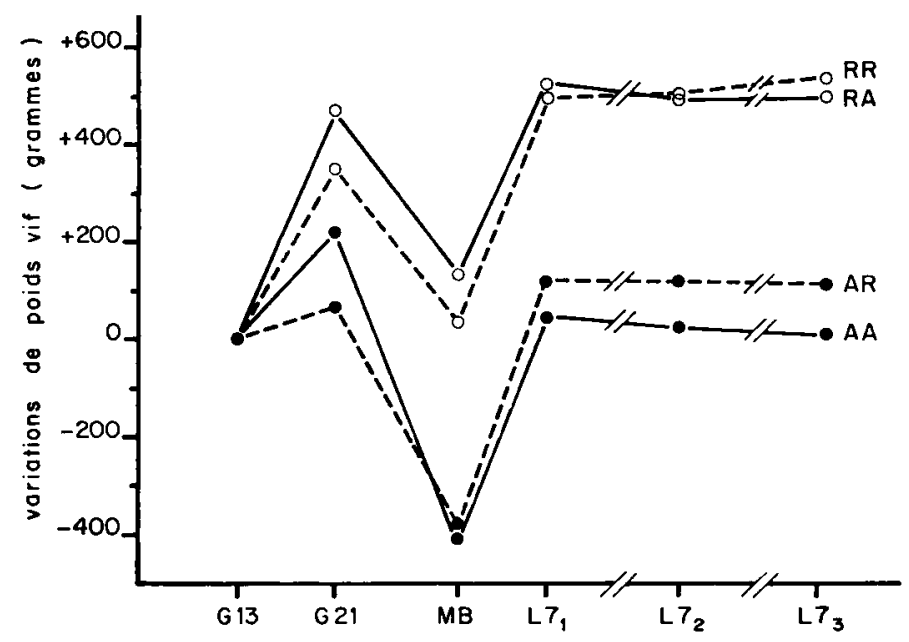

FIG. 1

Variations du poids vif des lapines des 4 lots expérimentaux entre la $1^{\text {re }}$ palpation positive (G13) et le $7^{e}$ jour suivant la $3^{c}$ mise bas $\left(L 7_{3}\right)$.

Live weight variations in does of the 4 experimental groups between the first positive pregnancy test (G13) and day 7 after the third kindling $\left(L 7_{s}\right)$.

D. Les premières mises bas (tabl. 2)

1. Effet du rationnement initial (lots $\mathrm{RR}+\mathrm{RA}$ vs $\mathrm{AR}+\mathrm{AA}$ )

Le nombre total de présentations au mâle nécessaire pour obtenir la fécondation n'est pas modifié par le rationnement. Les lapines qui étaient rationnées au moment de leur fécondation donnent des portées d'effectifs significativement réduits de 15 p. 100. Corrélativement, le poids moyen des lapereaux à la naissance est plus élevé. La mortalité entre la naissance et le sevrage n'est pas affectée par le traitement et l'effet constaté à la parturition sur la taille de la portée, se retrouve au sevrage. Il nous paraît important de remarquer que la limitation du poids des femelles, due au rationnement, toujours marquée à la palpation positive $(-10$ p. 100), a disparu à la mise bas ou 7 jours après cette dernière.

2. Effet du rationnement en fin de gestation (lots $\mathrm{RR}+\mathrm{AR} v s \mathrm{RA}+\mathrm{AA}$ )

L'effet du rationnement des lapines durant les deux tiers finaux de la g'station n'apparaît que sur la mortinatalité (augmentation) et pendant la lactation, sur la mortalité des lapereaux pour des causes autres que la mort de leur mère (réduction). 
TABlenu 2

Résultats des premières mises bas.

Results of the first kindlings.

\begin{tabular}{|c|c|c|c|c|c|c|c|c|}
\hline \multirow[t]{2}{*}{$\begin{array}{l}\text { Critères } \\
\text { Criteria }\end{array}$} & \multicolumn{4}{|c|}{$\begin{array}{l}\text { Lots } \\
\text { Groups }\end{array}$} & \multirow{2}{*}{$\begin{array}{l}\text { Coef. de } \\
\text { varia- } \\
\text { tion (1) } \\
(\%)\end{array}$} & \multicolumn{2}{|c|}{\begin{tabular}{|} 
Effet niveau \\
alimentaire \\
Effect of the level \\
of feeding
\end{tabular}} & \multirow[t]{2}{*}{$\begin{array}{l}\text { Inter- } \\
\text { action }\end{array}$} \\
\hline & $R R$ & RA & AR & $\mathrm{AA}$ & & ning & End & \\
\hline $\begin{array}{l}\text { Nombre total de mise bas } \\
\text { zéro nés vivants compris } \\
\text { (MBt) .................... } \\
\text { Total number of kindling } \\
\text { including zero live born } \\
(T K)\end{array}$ & 29 & 27 & 29 & 30 & - & - & - & 一 \\
\hline $\begin{array}{l}\text { Nombre de présentations/ } \\
\text { MB (2) } \ldots \ldots \ldots \ldots \ldots \ldots \\
\text { Number of services } / \text { TK }\end{array}$ & 1,28 & 1,59 & 1,38 & 1,20 & 55 & $\chi^{2} \mathrm{NS}$ & $\chi^{2}$ NS & $\chi^{2} \mathrm{NS}$ \\
\hline $\begin{array}{l}\text { Nombre de nés vivants/ } \\
\text { MBt ................. } \\
\text { Number of live born/TK }\end{array}$ & 8,03 & 8,67 & 9,86 & 9,90 & 34 & $\left(^{* *}\right)$ & NS & NS \\
\hline $\begin{array}{l}\text { Nombre de morts-nés/MBt } \\
\text { Number of dead born/TK }\end{array}$ & 0,79 & 0,56 & 1,17 & 0,57 & 247 & NS & NS & NS \\
\hline $\begin{array}{l}\text { Mortinatalité }(\%) \\
\text { Rate of stillbirths }\end{array}$ & 9,0 & 6,0 & 10,6 & 5,4 & 一 & $x^{2} \mathrm{NS}$ & $\chi^{2}(* *)$ & $\chi^{2} \mathrm{NS}$ \\
\hline $\begin{array}{l}\text { Nombe de mise bas avec au } \\
\text { moins } 1 \text { né vivant (MBv) } \\
\text { Kindling with at least } \\
1 \text { live born }(K l)\end{array}$ & 28 & 26 & 28 & 28 & - & - & - & - \\
\hline $\begin{array}{l}\text { Poids moyen des nés vi- } \\
\text { vants }(\mathrm{g}) \ldots \ldots \ldots \ldots \ldots \\
\text { Mean weight of live born }\end{array}$ & 54,6 & 54,5 & 51,2 & 48,7 & 14 & $(* *)$ & NS & NS \\
\hline $\begin{array}{l}\text { Nombre de sevrés/MBv .. } \\
\text { Number of weaned/Kl }\end{array}$ & 6,89 & 7,50 & 8,39 & 8,36 & 39 & $\left(^{*}\right)$ & NS & NS \\
\hline $\begin{array}{l}\text { Nombre total perdu entre } \\
\text { MB et sevrage ........ } \\
\text { Total loss between kindling } \\
\text { and weaning }\end{array}$ & 1,43 & 1,50 & 1,82 & 2,25 & 161 & NS & NS & NS \\
\hline
\end{tabular}


Tableau 2 (suite)

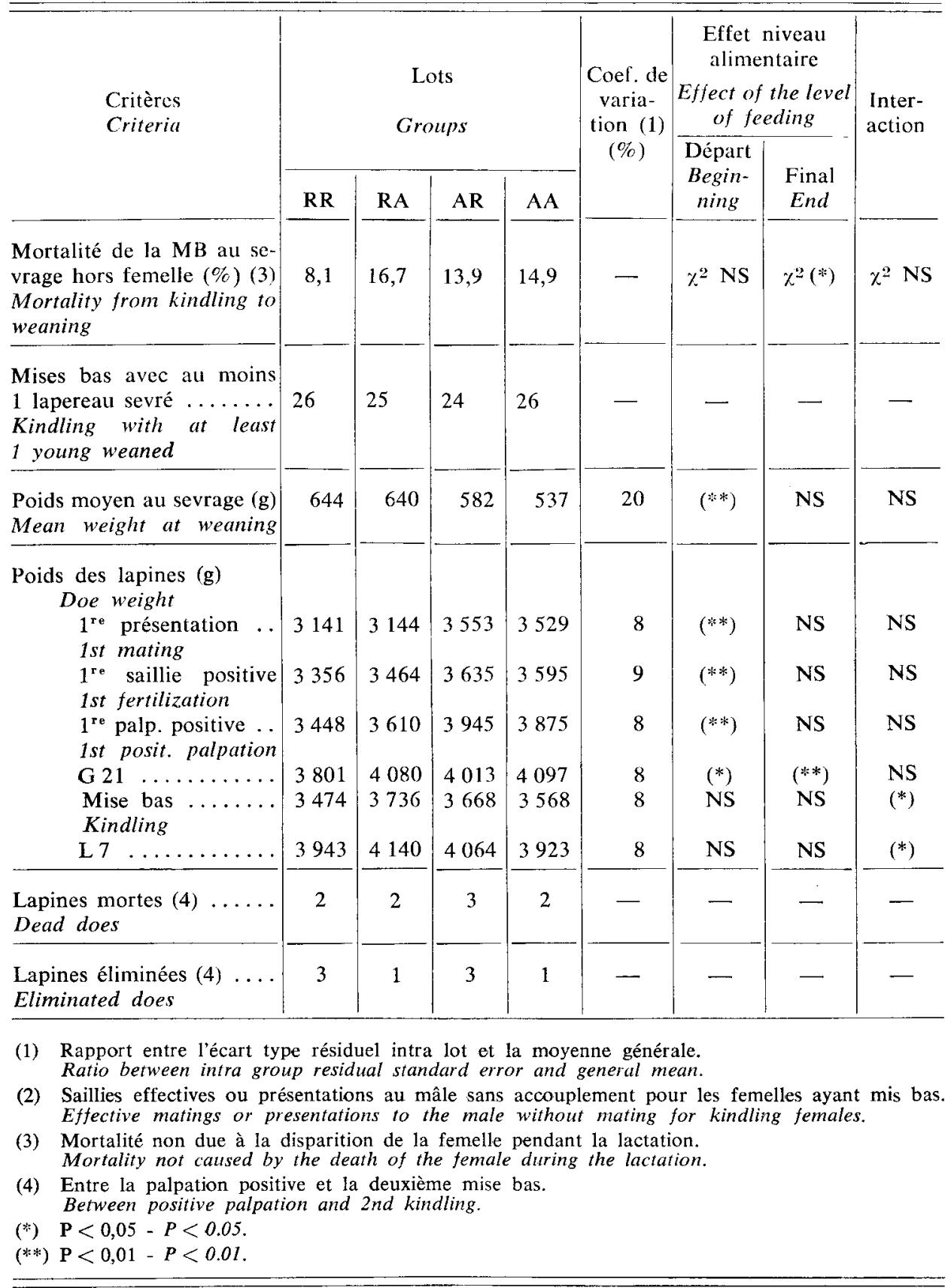


TABleaU 3

Résultats des deuxièmes mises bas.

Results of the second kindlings.

\begin{tabular}{|c|c|c|c|c|c|c|c|c|}
\hline \multirow[t]{2}{*}{$\begin{array}{l}\text { Critères } \\
\text { Criteria }\end{array}$} & \multicolumn{4}{|c|}{$\begin{array}{l}\text { Lots } \\
\text { Groups }\end{array}$} & \multirow{2}{*}{$\begin{array}{c}\text { Coef. de } \\
\text { varia- } \\
\text { tion }(1) \\
(\%)\end{array}$} & \multicolumn{2}{|c|}{\begin{tabular}{|c|} 
Effet niveau \\
alimentaire \\
Effect of the level \\
of feeding
\end{tabular}} & \multirow[t]{2}{*}{$\begin{array}{l}\text { Inter- } \\
\text { action }\end{array}$} \\
\hline & RR & RA & AR & AA & & $\begin{array}{c}\text { Départ } \\
\text { Begin- } \\
\text { ning }\end{array}$ & $\begin{array}{l}\text { Final } \\
\text { End }\end{array}$ & \\
\hline $\begin{array}{l}\text { Nombre total de mise bas } \\
\text { zéro nés vivants compris } \\
\text { (MBt) ................. } \\
\text { Total number of kindlins } \\
\text { including zero live born } \\
\text { (TK) }\end{array}$ & 24 & 25 & 24 & 27 & - & - & - & - \\
\hline $\begin{array}{l}\text { Nombre de présentations/ } \\
\text { MB (2) ............... } \\
\text { Number of services } / T K\end{array}$ & 1,42 & 1,72 & 1,63 & 1,74 & 60 & $\chi^{2}$ NS & $\chi^{2}$ NS & $\chi^{2} \mathrm{NS}$ \\
\hline $\begin{array}{l}\text { Intervalle entre } 1^{\text {re }} \text { et } 2^{c} \\
\text { mises bas (jours) } \ldots \ldots . . \\
\text { Interval between the } 1 \text { st } \\
\text { and } 2 \text { nd litter (days) }\end{array}$ & 47 & 49 & 48 & 50 & 7 & NS & NS & NS \\
\hline $\begin{array}{l}\text { Nombre de nés vivants/ } \\
\text { MBt .................. } \\
\text { Number of live born } / T K\end{array}$ & 9,46 & 10,04 & 9,50 & 9,96 & 34 & NS & NS & NS \\
\hline $\begin{array}{l}\text { Nombre de morts-nés/MBt } \\
\text { Number of dead born } / T K\end{array}$ & 0,86 & 1,00 & 1,29 & 0,96 & 225 & NS & NS & NS \\
\hline $\begin{array}{l}\text { Mortinatalité (\%) } \ldots \ldots \\
\text { Rate of stillbirths }\end{array}$ & 8,5 & 9,1 & 12,0 & 8,8 & 一 & $\chi^{2} \mathrm{NS}$ & $x^{2} \mathrm{NS}$ & $\chi^{2} \mathrm{NS}$ \\
\hline $\begin{array}{l}\text { Nombre de mise bas avec, } \\
\text { avec au moins } 1 \text { né vivant } \\
\text { (MBv) . . . . . . . . . } \\
\text { Kindling with at least } \\
\text { 1 live born (Kl) }\end{array}$ & 24 & 25 & 23 & 26 & - & - & - & - \\
\hline $\begin{array}{l}\text { Poids moyen naissance }(\mathrm{g}) \\
\text { Mean weight at birth }\end{array}$ & 61,0 & 55,6 & 56,5 & 59,7 & 12 & NS & NS & $(* *)$ \\
\hline $\begin{array}{l}\text { Nombre de sevrés } / \mathrm{MBv} \\
\text { Number of weaned } / \mathrm{Kl}\end{array}$ & 7,91 & 8,32 & 7,39 & 9,27 & 39 & NS & $\left(^{*}\right)$ & NS \\
\hline $\begin{array}{l}\text { Nombre total perdu entre } \\
\text { MB et sevrage ......... } \\
\text { Total loss between kindling } \\
\text { and weaning }\end{array}$ & 1,54 & 1,72 & 2,52 & 1,08 & 172 & NS & NS & NS \\
\hline
\end{tabular}


TABLEAU 3 (suite)

\begin{tabular}{|c|c|c|c|c|c|c|c|c|}
\hline \multirow[t]{2}{*}{$\begin{array}{l}\text { Critères } \\
\text { Criteria }\end{array}$} & \multicolumn{4}{|c|}{$\begin{array}{l}\text { Lots } \\
\text { Groups }\end{array}$} & \multirow{2}{*}{$\begin{array}{l}\text { Coef. } \\
\text { de } \\
\text { varia- } \\
\text { tion } \\
(1)\end{array}$} & \multicolumn{2}{|c|}{$\begin{array}{l}\text { Effet niveau } \\
\text { alimentaire } \\
\text { Effect of the } \\
\text { level of feeding }\end{array}$} & \multirow[t]{2}{*}{$\begin{array}{l}\text { Inter- } \\
\text { action }\end{array}$} \\
\hline & RR & RA & AR & AA & & $\begin{array}{l}\text { Départ } \\
\text { Begin- } \\
\text { ning }\end{array}$ & $\begin{array}{l}\text { Final } \\
\text { End }\end{array}$ & \\
\hline $\begin{array}{l}\text { Mortalité de la } \mathrm{MB} \text { au se- } \\
\text { vrage hors femelle }(\%)(3) \\
\text { Mortality from kindling to } \\
\text { weaning }\end{array}$ & 10,6 & 17,1 & 13,6 & 10,4 & - & $\chi^{2}$ NS & $\chi^{2} \mathrm{NS}$ & $x^{2}(*)$ \\
\hline $\begin{array}{l}\text { Mises bas avec au moins } \\
1 \text { lapereau sevré } \ldots . . . \\
\text { Kindling with at least } \\
1 \text { young weaned }\end{array}$ & 22 & 24 & 19 & 25 & 一 & - & - & 一 \\
\hline $\begin{array}{l}\text { Poids moyen au sevrage }(\mathrm{g}) \\
\text { Mean weight at weaning }\end{array}$ & 661 & 617 & 630 & 597 & 11 & NS & NS & NS \\
\hline $\begin{array}{l}\text { Différence de poids } \mathrm{L} 7 \mathrm{en}- \\
\text { tre les } 21^{\mathrm{re}} \text { portées }(\mathrm{g}) \ldots \\
\text { Weight differences between } \\
\text { the first two litters }\end{array}$ & $-0,2$ & $+0,8$ & $+2,4$ & $-22,6$ & (5) & NS & NS & NS \\
\hline $\begin{array}{l}\text { Femelles mortes (4) ... } \\
\text { Dead does }\end{array}$ & 1 & 1 & 3 & 0 & - & - & - & - \\
\hline $\begin{array}{l}\text { Femelles éliminées (4) .. } \\
\text { Eliminated does }\end{array}$ & 1 & 1 & 1 & 1 & - & - & - & - \\
\hline
\end{tabular}

(1) Rapport entre l'écart type résiduel intra lot et la moyenne générale. Ratio between intra group residual standard error and general mean.

(2) Saillies effectives ou présentations au mâle sans accouplement pour les femelles ayant mis bas. Effective matings or presentations to the male without mating for kindling females.

(3) Mortalité non due à la disparition de la femelle pendant la lactation. Mortality not caused by the death of the female during the lactation.

(4) Entre deuxième mise bas et troisième mise bas. Between 2nd kindling and 3rd kindling.

(5) Coefficient de variation sans signification pour une moyenne générale égale à $-5,6 \mathrm{~g}$ et un écart type de $215 \mathrm{~g}$.

V.C. without significance for a general mean of $-5.6 \mathrm{~g}$ and a standard error of $215 \mathrm{~g}$.

(*) $\mathbf{P}<0,05-P<0.05$.

(**) $\mathrm{P}<0,01-P<0.01$. 
Par contre, il n'y a pas d'altération du poids moyen des lapereaux à la naissance. Le poids des lapines lors de la mise bas ou à $L$ 7 ne diffère pas en fonction du rationnement en fin de gestation, alors qu'il était plus faible à $\mathrm{G} 21$ pour les lapines rationnées, par rapport à celui des lapines alimentées à volonté à cette période. Cette situation était d'ailleurs due exclusivement aux femelles du lot RR.

\section{Interaction entre les deux périodes de rationnement (lots $\mathrm{RR}+\mathrm{AA}$ vs $\mathrm{RA}+\mathrm{AR}$ )}

Aucun effet significatif du changement de niveau d'alimentation n'est observé sur les portées; mais les femelles qui ont subi ce changement, ont, à partir de la mise bas, un poids significativement plus élevé que celui des femelles maintenues au même niveau d'alimentation.

\section{E. Les deuxièmes mises bas (tabl. 3)}

Les effets, sur les lapereaux, du rationnement initial ou final, observés durant la première portée ne le sont plus à l'occasion des deuxièmes portées. Par contre, les lapines qui ont subi un changement de niveau d'alimentation (lots RA et AR) ont donné naissance en deuxième mise bas à des lapereaux significativement plus légers $(\mathrm{P}<0,01)$, sans aucune altération de la taille de la portée. Parallèlement, la mortalité entre naissance et sevrage a été significativement plus élevée pour ces deux lots.

Par ailleurs, les lapines du lot AR ont perdu, en moyenne, plus de lapereaux entre la naissance et le sevrage de la deuxième portée. Cette mortalité est en grande partie expliquée par la mort de 3 femelles au cours de la lactation. En conséquence, le nombre moyen de lapereaux sevrés est significativement plus faible pour le groupe de lapines RR + AR que pour celui des femelles AA + RA. Par contre, la mortalité entre naissance et sevrage n'est pas reliée au niveau de rationnement des lapines au cours de leur $1^{\text {re }}$ gestation.

Le nombre de présentations au mâle, nécessaire pour obtenir les deuxièmes portées, n'a pas été affecté par les niveaux d'alimentation avant la première mise bas; en conséquence, les intervalles entre les deux premières mises bas sont proches les unes des autres pour les 4 lots. Enfin, il nous semble important de souligner la remarquable constance d'une portée à l'autre du poids des lapines, mesuré 7 jours après la mise bas, quel que soit le lot expérimental.

\section{F. Résultat global après 22 semaines d'expérience}

Dans leur majorité, les lapines vivantes en fin d'essai ont réalisé trois portées au moment de l'arrêt de l'expérimentation; toutefois, 10 des 91 lapines vivantes au moment des troisièmes mises bas n'ont fait que 2 portées dans les limites de l'essai (tabl. 1). Pour cette raison, les résultats des troisièmes mises bas n'ont pas été analysés séparément. Les performances globales présentées ci-après intègrent donc le facteur temps dans la comparaison de la productivité des lapines des 4 lots (tabl. 4).

Les 31 lapines mises en place dans le lot AA ont permis le sevrage de 636 lapereaux à partir de 76 mises bas; ceci représente un accroissement de la production 
TABLEAU 4

Résultats globaux en fin d'expérimentation.

Global results at the end of the experiment.

\begin{tabular}{|c|c|c|c|c|c|c|c|c|}
\hline \multirow[t]{2}{*}{$\begin{array}{l}\text { Critères } \\
\text { Criteria }\end{array}$} & \multicolumn{4}{|c|}{$\begin{array}{l}\text { Lots } \\
\text { Groups }\end{array}$} & \multirow{2}{*}{$\begin{array}{c}\text { Coef. de } \\
\text { varia- } \\
\text { tion } \\
(\%)\end{array}$} & \multicolumn{2}{|c|}{\begin{tabular}{|} 
Effet niveau \\
alimentaire \\
Effect of the level \\
of feeding
\end{tabular}} & \multirow[t]{2}{*}{$\begin{array}{l}\text { Inter- } \\
\text { action }\end{array}$} \\
\hline & RR & RA & AR & AA & & $\begin{array}{l}\text { Départ } \\
\text { Begin- } \\
\text { ning }\end{array}$ & $\begin{array}{c}\text { Final } \\
\text { End }\end{array}$ & \\
\hline $\begin{array}{l}\text { Nombre de femelles au } \\
\text { départ } \ldots \ldots \ldots \ldots \ldots \ldots \ldots \\
\text { Number of females at the } \\
\text { beginning }\end{array}$ & 31 & 31 & 31 & 31 & - & - & - & - \\
\hline $\begin{array}{l}\text { Nombre de mises bas } \ldots \\
\text { Number of kindlings }\end{array}$ & 71 & 70 & 69 & 76 & - & 一 & - & 一 \\
\hline $\begin{array}{l}\text { Nombre de nés vivants } \\
\text { Number of live born }\end{array}$ & 624 & 674 & 664 & 760 & - & 一 & - & - \\
\hline $\begin{array}{l}\text { Nés vivants/mises bas } \\
\text { Live born/kindlings }\end{array}$ & 8,79 & 9,63 & 9,62 & 10,00 & 33 & NS & NS & NS \\
\hline $\begin{array}{l}\% \text { de morts-nés } \ldots \ldots \ldots \\
\% \text { of live born }\end{array}$ & 8,91 & 7,67 & 10,63 & 6,98 & - & NS & $(*)$ & NS \\
\hline $\begin{array}{l}\text { Nombre de sevrés } . . . \ldots \\
\text { Number of weaned }\end{array}$ & 529 & 539 & 530 & 636 & - & - & - & - \\
\hline $\begin{array}{l}\text { Sevrés par mises bas .... } \\
\text { Number of weaned young } / \\
\text { kindling }\end{array}$ & 7,45 & 7,70 & 7,68 & 8,37 & 45 & NS & NS & NS \\
\hline $\begin{array}{l}\text { Mortalité entre mise bas } \\
\text { et sevrage hors femelle } \\
\text { (\%) (1) ............. } \\
\text { Mortality between kindling } \\
\text { and weaning }\end{array}$ & 10,0 & 17,5 & 14,4 & 12,8 & - & NS & $\left(^{*}\right)$ & $(* *)$ \\
\hline $\begin{array}{l}\text { Poids de portée au se- } \\
\text { vrage }(\mathrm{g}) \ldots \ldots \ldots \ldots \ldots \\
\text { Litter weight at weaning }\end{array}$ & 5141 & 5004 & 5199 & 4918 & 24 & NS & NS & NS \\
\hline $\begin{array}{l}\text { Fonte du cheptel en fin } \\
\text { d'essai }(\%) \ldots \ldots \ldots \ldots \\
\text { Stock reduction at the end } \\
\text { of the experiment }\end{array}$ & 29 & 42 & 45 & 32 & - & NS & NS & NS \\
\hline
\end{tabular}

(1) Mo:talité non due à la disparition de la femelle pendant la lactation. Mortality not caused by the death of the female during lactation. 
globale de 19,4 p. 100 par rapport à celle obtenue par les 31 lapines initiales de chacun des 3 autres lots. Cet écart correspond à un nombre de mises bas légèrement plus élevé, associées à une bonne prolificité mais sans accroissement du poids moyen des portées au sevrage. Sur l'ensemble des portées nées, les femelles des lots RA et AR, ayant changé de niveau d'alimentation, ont perdu une plus forte proportion des lapereaux entre la naissance et le sevrage. Cette mortalité n'est pas apparue dès la première portée, mais s'est manifestée en deuxième portée avec suffisamment d'intensité pour influer négativement sur les résultats de l'ensemble de la période expérimentale.

\section{G. Fonte du cheptel}

Il semble important de rappeler que 3 femelles rationnées ont été éliminées pour stérilité avant la première portée. Par la suite, cette cause d'élimination a été retenue 3 autres fois pour les lapines rationnées durant la fin de leur croissance (lot RR $+\mathrm{RA}$ ). Dans le cas des lapines nourries à volonté avant la première palpation (lot AA + AR), l'élimination pour stérilité n'a été retenue qu'une seule fois $(\mathrm{P}<0,05)$.

Le rationnement en fin de gestation tend à accroître le nombre de femelles qu'il a fallu éliminer avant la deuxième mise bas : 6 vs 2. En deuxième portée, les femelles éliminées sont également réparties entre les 4 lots (tabl. 3). Les femelles mortes au cours de leur première ou de leur deuxième lactation avaient, à $L$, un poids vif comparable à celui des autres lapines, quel que soit le lot expérimental. Au total, avant la troisième mise bas, 16 lapines (28 p. 100 des lapines initiales) avaient disparu par décès ou élimination dans les 2 lots rationnés en fin de gestation $(\mathrm{RR}+\mathrm{AR})$, alors que leur nombre est moitié $(\mathrm{P}<0,10)$ pour les 2 autres lots (8 lapines, soit 14 p. 100). Entre la troisième mise bas et le sevrage des portées correspondant, 1 à 2 lapines ont été perdues dans chacun des lots. Par contre, il convient de signaler la forte élimination lors du troisième sevrage, pour les lapines des 2 lots qui étaient nourries à volonté à la fin de la première gestation : 10 vs 3 pour les 2 autres lots $(\mathrm{P}<0,10)$. Dans le lot $\mathrm{AA}$, les 6 lapines qui ont dû être éliminées l'ont toutes été pour abcès mammaire, alors que cette cause n'a été retenue que 2 fois pour les 20 lapines éliminées dans l'ensemble des 3 autres lots $(P<0,01)$. De plus, en fin d'essai, les femelles AA avaient un plus mauvais état sanitaire apparent que les femelles des 3 autres lots : 6 femelles AA sur 21 vivantes avaient des «problèmes sanitaires apparents" (dont 3 cas d'abcès mammaire) alors que ce nombre est de $1 / 17$ et $1 / 18$ pour les lots RA et AR. Toutefois, 5 femelles sur 22 avaient aussi des "problèmes sanitaires» dans le lot $R R$, mais avec un degré de gravité moins grand que pour le lot AA.

Enfin, il nous paraît opportun de rapprocher la plus forte mortinatalité observée dans le lot AR de la forte mortalité des femelles de ce lot : double de celle observée dans les 3 autres lots (tabl. 1).

\section{Discussion}

Il est important, dans ce type d'étude sur les lapines reproductrices, de bien préciser toutes les conditions expérimentales. Ainsi, la fréquence de nettoyage du 
matériel ou le rythme de reproduction imposé sont susceptibles d'avoir une incidence sur les phénomènes morbides et les performances des animaux (Suridiau, Perrier \& Plassier, 1984). De plus, à l'occasion de cette expérimentation, il nous paraît important de souligner que le calcul et l'interprétation des phénomènes morbides posent des problèmes d'ordre méthodologique aux expérimentateurs. L'inclusion ou l'exclusion d'un petit groupe d'animaux dans une catégorie comportant un grand nombre d'individus peut modifier complètement l'interprétation statistique des chiffres et par là, la recherche des causes potentielles de morbidité. Ainsi, le comportement morbide de quelques femelles (avortement, portées entières de morts nés, mort de la femelle avant le sevrage) peut biaiser totalement l'analyse du taux de mortalité des lapereaux en raison de l'effet démultiplicateur de la taille de la portée ; à titre d'exemple, si dans un groupe de 100 lapines perdant 4 à 5 p. 100 de leurs lapereaux, on ajoute seulement 5 femelles perdant la totalité de leur portée, la mortalité du nouveau groupe de 105 femelles est numériquement double de celle des 100 lapines initiales. Suivant que l'on retient l'un ou l'autre mode de calcul, on recherchera la cause de mortalité principale soit chez les lapereaux eux-mêmes, soit chez leur mère, or les mécanismes mis en jeu ne sont pas les mêmes (Coudert, Viard-Drouet \& Provot, 1984).

Indépendamment de l'effet des traitements, la production des lapines et la fonte du cheptel observécs dans notre expérimentation sont comparables dans leur importance et leur chronologie à celles enregistrées antérieurement sur le même élevage (COUdert, 1980 ; Coudert \& Lebas, 1982). En particulier, il nous semble important de souligner le nombre élevé de lapereaux nés vivants par portée dans ces expérimentations : 9,52 en moyenne dans le cas présent (10,43 nés totaux), alors qu'il n'est que de 7,47 par exemple dans des élevages cunicoles rationnels du «terrain » (DE LA Bouillerie et al., 1980). En effet, les dépenses de l'organisme des lapines, tant pour la gestation que la lactation, sont fortement corrélées avec la taille de leur portée, et nous pensons que cela influence nettement l'évolution de leur productivité et des phénomènes morbides.

Le rationnement initial des lapines a un effet beaucoup plus prolongé sur leur poids vif que le rationnement final. Pour faciliter l'interprétation, nous avons reporté sur la figure 1 les variations de poids des lapines à partir de la palpation positive à $G 13$, c'est-à-dire du changement éventuel de niveau d'alimentation. Il y apparaît clairement que les variations de poids des lapines sont très fortement conditionnées par le rationnement initial : les courbes des lots RR et RA sont pratiquement superposćes et il en est de même des courbes des lots AA et AR. Par ailleurs, les lapines rationnées jusqu'à $G 13$ (RR et $R A$ ) ont un gain de poids entre $G 13$ et $G 21$ (milieu de la première gestation) beaucoup plus important que celui observé pour les lapines nourries à volonté jusqu'à $G$ 13. Le gain de poids observé chez ces dernières correspond à peu de chose près à la variation de poids de l'utérus et de son contenu. En effet, chez la lapine adulte, le gain de poids de l'utćrus plein a été estimé en moyenne à $182 \mathrm{~g}$ entre G 10 et G 21 par LEBAS (1975), et dans notre cas, le poids des femelles des lots AR + AA a augmenté de $145 \mathrm{~g}$ entre $\mathrm{G} 13$ et G 21 . En conséquence, on peut penser que le gain de 412 g entre $G 13$ et $G 21$ observé chez les femelles RR + RA correspond en majeure partie à un gain propre de la lapine. Cette constatation implique que le rationnement initial a modifié de manière durable - au moins la durée nécessaire à finir la gestation - l'état physiologique des lapines. Tout se passe comme si l'état gravidique permettait aux lapines de réaliser une croissance compensatrice, même à niveau d'alimentation constant (lot RR), 
ce qui veut dire que les aliments ont été mieux valorisés. En effet, le rattrapage pondéral des lapines antérieurement rationnées par rapport aux femelles antérieurement nourries à volonté ne peut correspondre à une simple rétention d'eau ou à un dépôt de gras puisqu'il est définitivement acquis. Effectivement, nous n'observons plus d'écart de poids entre les lots de femelles à partir du début de la $1^{\text {re }}$ lactation. Pour expliquer ce brusque accroissement de poids, on peut faire l'hypothèse que l'hyperplasie cellulaire freinée par la restriction alimentaire (DURAND, Fauconneau \& Penot, 1969) reprenne lors de la gestation sous l'action de la progestérone (Bressi: 1953). Notons en outre que le poids des lapines mesuré 7 jours après la mise bas est une bonne estimation du poids adulte de nos lapines puisqu'il ne varie pas d'une portée à l'autre.

Van den Broeck \& Lampo (1979), Hulot, Mariana \& Lebas (1982), avaient constaté des difficultés pour obtenir les premiers accouplements lorsque les jeunes lapines étaient rationnées en fin de croissance. Bien que n'étant pas significatifs, nos résultats vont dans le même sens. On peut faire l'hypothèse que la réalisation d'un choc lumineux, avant la première présentation au mâle, pratique favorisant l'acceptation de l'accouplement (LeFEvre \& Morer, 1978), a atténué l'effet du rationnement. Par contre, on peut exclure un effet de la maturité sexuelle puisque les jeunes lapines ont été présentées pour la première fois au mâle à un âge (123 jours) où l'effet du rationnement alimentaire ne se fait plus sentir sur le comportement sexuel (Hucot, Mariana \& Lebas, 1982). Par ailleurs, et contrairement aux observations de VAN DEN BROIEcK \& LAMPo (1979), nous avons enregistré une nette réduction du nombre de lapereaux nés vivants. Hulot, Mariana \& Lebas (1982) avaient bien enregistré une réduction du nombre d'ovules pondus par des jeunes femelles du même âge et ils avaient attribué cette différence au poids des mères $:+1$ ovule pour environ $400 \mathrm{~g}$ de poids vif supplémentaire lors de la saillie fécondante. Or, dans notre cas. l'écart de poids vif n'est que de $205 \mathrm{~g}$ pour une réduction de 1,7 lapereaux nés. Toutefois, on doit remarquer la plus forte prolificité de la souche (hybride) que nous avons utiliséc : 10,7 nés totaux, alors que Hulot, Mariana \& Lebas (1982) n'enregistrent que 8,3 à 9,2 ovules pondus et que, pour VAN DEN BroEcK \& LAMPo (1979), la taille de portée à la naissance n'était que de 8,6 à 9,2 nés totaux. Il est possible que le rationnement précoce n'affecte la prolificité que lorsque les potentialités sont très élevées. L'existence d'un désaccord entre auteurs sur l'effet du rationnement des jeunes femelles sur leur prolificité existe pour d'autres espèces comme le porc (Duee \& Etienne, 1974 ; Lodge \& McPherson, 1961). Mais dans tous les cas, lorsqu'il y a un effet du rationnement sur la prolificité, il se traduit par une réduction de la taille de la portée. Dans notre essai, pour tenter d'expliquer cet effet néfaste, nous pouvons émettre l'hypothèse que le fort gain de poids des lapines des lots RR et RA, observé à partir de G 13, se soit réalisé aux dépens des embryons et ait accru la mortalité embryonnaire chez ces lapines en fin de croissance. Un accroissement de la mortalité embryonnaire dû au rationnement des jeunes mères au moment de la saillie a d'ailleurs été décrit par PIKE \& BoAz (1972) chez la truic primipare. Le rationnement initial des lapines n'a pas modifié la mortalité des lapereaux, ni l'importance de la fonte du cheptel. Par contre, il a augmenté significativement le pourcentage de femelles éliminées pour "stérilité ". Ce dernicr fait mérite d'être rapproché des difficultés observées pour obtenir les premières gestations.

Le rationnement des lapines pendant les deux tiers finaux de la premic̀re gestation n'a pas de conséquence majeure sur l'évolution du poids des femelles. Il ne fait que moduler l'ampleur des variations liées au niveau d'alimentation antérieur (fig. 1). 
Par contre, ce rationnement en fin de première gestation accroît significativement le pourcentagc de morts nés lors de la première mise bas ; à l'inverse, il permet une réduction de la mortalité pendant la lactation. En fait, cette réduction de la mortalité n'existe que lors de la comparaison des lots RA et RR (16,7 p. 100 vs 8,1 p. 100). LEßsAS (1975) a déjà observé chez les lapines rationnées pendant la gestation, une telle réduction de la mortalité au cours de la lactation. Il a démontré qu'elle est liée à une plus forte production laitière les 8 jours suivant la mise bas chez les lapines rationnées en gestation, puis nourries à volonté après la mise bas. Nous pouvons donc penser que les lapines du lot $R R$ avec une taille de portée relativement faible à la naissance $(8,03)$, ont pu accroitre leur production laitière par lapereau par rapport à celle des lapines RA ayant des portées comparables $(8,67$ nés vivants par mise bas) ou par rapport à celle des lapines AA plus prolifiques. Par contre, les lapines AR avec 9,86 lapereaux nés vivants ont probablement atteint le maximum de leur capacité de production (Lebas, 1969) et n'ont donc pas pu accroître sensiblement leur production laitière par lapereau, comparativement aux femelles AA ayant la même prolificité ; et en conséquence, le taux de mortalité des lapereaux pendant la lactation a été comparable pour les deux lots $\mathrm{AR}$ et $\mathrm{AA}$, alors qu'il a été réduit par le rationnement final dans le lot RR par rapport au lot RA.

Le poids significativement plus élevé observé immédiatement ou 7 jours après la première mise bas chez les lapines ayant changé de niveau d'alimentation à $G 13$ n'est sans doute que la conséquence des écarts de poids liés à l'échantillonnage. En effet, à $G$ 13, donc avant le changement de niveau alimentaire, ces lapines avaient déjà un poids plus élevé que celui des femelles correspondantes ne devant pas changer de niveau d'alimentation. Pour le groupe des femelles RA et AR, le poids moyen des lapereaux à la naissance de la deuxième portée est significativement plus faible que celui des lapereaux des femelles AA et RR. Ceci ne peut pas être expliqué par la taille de la portée à la naissance. Par contre, nous pouvons faire l'hypothèse que ce poids réduit est une des conséquences d'un mauvais état général des lapines, qui se traduit en fin d'expérimentation par une fonte du cheptel plus forte. Ces deux phénomènes conjugués expliquent probablement la plus forte mortalité observée entre naissance et sevrage pour les lots RA et AR, aussi bien en deuxième portée qu'au bilan global de l'expérimentation.

Dans le cas des lapines AA, nous avons observé, en fin d'essai, d'une part une forte fréquence des lapines éliminées pour abcès mammaire et d'autre part un état sanitaire plus mauvais que pour les lapines des 3 autres lots. Ces deux observations sont à rapprocher du fait que ces lapines sont aussi celles qui ont allaité le plus grand nombre de lapereaux.

\section{Conclusion}

En conclusion de cette expérimentation, on peut retenir que le rationnement des jeunes femelles en fin de croissance n'altère pas leur poids adulte. En revanche, il diminue la production de lapereaux sevrés, essentiellement en raison d'une réduction de la taille de la première portée.

Le rationnement au cours des deux derniers tiers de la première gestation entraîne une fonte plus précoce du cheptel. Enfin, le changement de niveau d'alimen- 
tation au cours de la première gestation a des conséquences néfastes en cieuxième ct troisième portée, d'une part sur la mortalité des lapereaux allaités et d'autre part sur la fonte du cheptel. Globalement, les lapines nourries en permanence à volonté ont donné la plus forte production.

\section{Remerciements}

Les autcurs tiennent à remercier le personnel des Unités de Pathologie et d'Informatique du Domaine I.N.R.A. du Magneraud pour leur participation à l'exécution de cette expérimentation.

\section{Summary \\ Productivity and morbidity of breeding does. 1. - Effects of feed restriction before and during first gestation}

A total of 124 crossbred does I.N.R.A. A 1067 (New Zealand White $\times$ Californian) were distributed into four treatments of the factorial scheme $2 \times 2$ corresponding to a restricted $(150 \mathrm{~g} / \mathrm{d})$ or an ad libitum feeding (ie. $200 \mathrm{~g} / \mathrm{d}$ ) from the age of 79 days to first kindling with or without inversion of the level of feeding from day 13 of pregnancy (positive pregnancy test). Does were mated 10 days after each kindling.

The initial feed restriction level reduced by $11 \mathrm{p} .100$ the live weight measured at first mating. Conversely, it did not modify the adult weight estimated on the basis of the weight 7 days after each kindling. The feed restriction level in late 1 st pregnancy had only a transitory effect on the doe live weight.

The litter size of does restricted at 1st fertilization was significantly smaller : 8.35 vs 9.88 live born/kindling. This was no more observed in the next kindlings. Feed restriction during the last two thirds of first gestation did not significantly affect the mean weight of the young: $52.9 \mathrm{~g}$ vs $51.6 \mathrm{~g}$ for does fed ad libitum. However, this feed restriction led to an earlier stock reduction. Moreover, the modification of the level of feeding during the 1st pregnancy had unfavourable effects, i.e. a higher mortality rate of the suckled young from the 2 nd and 3rd litters and a stock reduction.

During the 22 weeks of experiment between 1st mating and last weaning, the 31 does fed ad libitum weaned 636 young versus 529 to 539 in the other 3 groups.

Key words : rabbit, female, gestation, feeding restriction, morbidity.

Regu en juin 1984.

Accepté en octobre 1984.

\section{Références bibliographiques}

Bachacou J., Masson J.P., Millier C., 1981. Le programme ANVAR in Manuel de la programmathèque statistique Amance 81, 341-369, I.N.R.A. éd., Paris.

Bresse G., 1953. Morphologie et physiologie animales. Librairie Larousse, éd. Paris.

Coudert P., 1980. Pathologie et conduite de l'élevage de lapines reproductrices. Le Point Vétérinaire, $10(50), 61-65$. 
Coudert P., Lebas F., 1982. Incidence de divers facteurs pathologiques et nutritionnels survenant pendant la croissance, sur le devenir des reproductrices. $3^{\prime s}$ Journées de la Recherche cunicole en France, I.T.A.V.I., éd. Paris, Communication 33.

Coudert P., Viard-Drouet F., Provot F., 1984. Pathologie des lapines reproductrices. Etude descriptive comparative des phénomènes morbides observés lors de la reproduction de deux souches pures de lapin. Ann. Rech. Vét., 15, 535-541.

De La Boulllerie R., Camps J., Ugo 1., Beaujour-Bourget H., 1980. Enquête technique sur la production de lapins de chair en élevages rationnels en France, Espagne et Italie. Mémoire $2^{\prime}$ Congrès Mondial de Cuniculture, Barcelone, avril 1980, Vol. I, 434-445.

Duee P.H., Erienne M., 1974. Influence de l'alimentation pendant la croissance de la truie sur la maturité sexuelle et les performances de reproduction : Résultats préliminaires. Journées Rech. porcine en France, 43-47, I.N.R.A., I.T.P. éd., Paris.

Durand G., Fauconneau G., Penot E., 1969. Répartition des cellules entre les tissus du rat adulte préalablement soumis à une sous-nutrition énergétique temporaire à deux stades de la croissance. Ann. Biol. anim. Biochim. Biophys., 9, 55-73.

Hulot F., Mariana J.C., Lebas F., 1982. L'établissement de la puberté chez la lapine (folliculogénèse et ovulation). Effet du rationnement alimentaire. Reprod. Nutr. Dévelop., 22, 439-453.

LEBAS F., 1969. Alimentation lactée et croissance pondérale du lapin avant sevrage. $A n n$. Zootech., 18, 197-208.

Lebas F., 1975. Etude chez la lapine de l'influence du niveau d'alimentation durant la gestation. 1) Sur les performances de reproduction. Ann. Zootech., 24, 267-279.

Lefevre B., MoreT B., 1978. Influence d'une modification brutale de l'environnement sur l'apparition de l'ocstrus chez la lapine nullipare. Ann. Biol. anim. Biochim. Biophys., 18, $695-698$.

Lodge G.A., McPherson R.M., 1961. Level of feeding during early life and the subsequent reproductive performance of sows. Anim. Prod., 3, 19-28.

PIKE I.H., BoAz T.G., 1972. The effect of condition at service and plane of nutrition in early pregnancy in the sow. I) Uterine and extra-uterine changes. Anim. Prod., 15, 147-155.

Surdeau Ph., Perrier G., Plassier J.L., 1984. Réponse biologique des lapines adoptant différents rythmes de reproduction. $3^{r}$ Congrès Mondial de Cuniculture, Rome, avril 1984 , Vol. II, 104-116, ASIC éd., Rome.

Van den Broeck L., Lampo P., 1977. Influence de 3 niveaux d'alimentation des lapines futures reproductrices sur l'ardeur sexuelle et la fertilité à 4 mois. Ann. Zootech., 26, 565-574.

Van den Broeck L., Lampo P., 1979. Influence de l'âge au premier accouplement sur la fertilité des jeunes lapines et leurs performances en première portée. Ann. Zootech., 28, 443-452.

Viard-Drouet F., Provot F., Covdert P., 1984. Evolution des paramètres plasmatiques chez des lapines repoductrices en fonction de l'état physiologique et du rationnement alimentaire. Ann. Rech. Vét., 15, 417-424. 